\title{
Efeitos do conhecimento de resultados autocontrolado na aprendizagem motora
}

\author{
Gabriela Moara Ferreira ${ }^{1}$ \\ Maicon Rodrigues Albuquerque 2, 3 \\ Natália Fontes Alves Ambrósio ${ }^{4}$ \\ Alessandro Teodoro Bruzi ${ }^{5}$ \\ Leandro Ribeiro Palhares ${ }^{1}$ \\ ${ }^{1}$ Departamento de Ciências Biológicas e da Saúde, \\ Universidade Federal dos Vales do Jequitinhonha e Mucuri, Diamantina, MG, Brasil \\ 2 Instituto Superior de Educação Anísio Teixeira - Fundação Helena Antipoff, Ibirité, MG, Brasil \\ ${ }^{3}$ Faculdade de Ciências Humanas, Sociais e da Saúde, Universidade FUMEC, Belo Horizonte, MG, Brasil \\ ${ }^{4}$ Escola de Educação Física e Esporte, USP - Universidade de São Paulo, São Paulo, SP, Brasil \\ ${ }^{5}$ Departamento de Educação Física, Universidade Federal de Lavras, MG, Brasil
}

\begin{abstract}
Resumo: O objetivo do presente estudo foi analisar três diferentes estratégias de fornecer conhecimento de resultados (CR): autocontrolado, yoked pareado por tentativa e yoked pareado pela frequência média total de CR, na prática aleatória. A amostra foi constituída por 45 voluntários universitários, distribuídos em três grupos ( $n=15$ sujeitos). A tarefa consistiu em pressionar as teclas 2, 4, 8 e 6 do teclado numérico de um computador, em três diferentes tempos alvo $(700,900$ e $1100 \mathrm{~ms})$, praticados aleatoriamente. $O$ experimento constou de fase de aquisição e testes de retenção e transferência atrasados. $A$ análise dos dados foi realizada por meio da ANOVA e não foi constatada diferença significativa entre os grupos. Os resultados demonstraram que não houve efeito das frequências autocontroladas para a aprendizagem motora quando se utiliza a prática aleatória. Além disso, uma nova possibilidade de parear o grupo autocontrolado foi apresentada.
\end{abstract}

Palavras-chave: Aprendizagem motora. Conhecimento de resultados. Frequência autocontrolada. Prática aleatória.

\section{Effects of self-controlled knowledge of results in motor learning}

Abstract: The purpose of this study was to examine three different strategies to provide knowledge of results $(K R)$, yoked paired by trial and yoked paired by average of total frequency of $K R$ in random practice. The sample was composed by 45 volunteers, distributed into three groups ( $n=15$ subjects). The task consisted of press three keys, 2, 4, 8 and 6 in the numeric keypad of the computer in three different total time $(700,900$ and $1100 \mathrm{~ms})$ in random practice. The experiment consisted of acquisition phase and delay retention and transfer test. The data analysis was conducted by ANOVA and the results were not demonstrated difference between groups. The results showed no effect of self-controlled frequency of KR to the motor learning when using random practice. In addition, a new possibility to be used the yoked group was presented.

Keywords: Motor learning. Knowledge of results. Self-controlled frequency. Random practice.

\section{Introdução}

Segundo Schmidt e Lee (2005), aprendizagem motora refere-se a um conjunto de processos associados com a prática e experiência que levam a mudanças relativamente permanentes no desempenho. Sendo que para Manoel (1999), a aprendizagem motora ocorre com auxílio de prática sistemática e feedback. No presente artigo, a Aprendizagem Motora será compreendida como as mudanças relativamente permanentes no comportamento motor, influenciadas pela prática sistematizada e informações sobre a prática. Em relação à informação, o conhecimento de resultados (CR) é uma forma de feedback extrínseco de grande relevância para a aprendizagem de habilidades motoras, informando o executante sobre o resultado do seu movimento em relação à meta da tarefa (SCHMIDT, 1975; CHIVIACOWSKY et al., 2007).

Diversos estudos que buscaram investigar os efeitos do $\mathrm{CR}$ mostraram que quanto mais frequente fosse o fornecimento de CR, melhor seria $O$ seu efeito na aprendizagem de 
habilidades motoras (ADAMS, 1971; BILODEAU; BILODEAU, 1958). Entretanto, Salmoni et al. (1984) realizaram uma revisão sobre os estudos de CR e apontaram críticas aos resultados encontrados, devido principalmente ao fato que os estudos anteriores não realizarem testes em seus delineamentos, que são responsáveis por distinguir os efeitos transitórios daqueles relativamente permanentes da aprendizagem.

Com a inserção dos testes de retenção e transferência, no sentido de verificar os efeitos da frequência relativa de $C R$ na aquisição de habilidades motoras (WULF; SCHMIDT, 1989; WINSTEIN; SCHMIDT, 1990), os resultados têm mostrado superioridade de grupos com frequências reduzidas quando comparados àqueles com altas frequências (WULF; SCHMIDT, 1989; WINSTEIN; SCHMIDT, 1990; OLIVEIRA et al., 2009).

Estes resultados parecem confirmar duas diferentes hipóteses: A hipótese denominada de orientação (guidance hypothesis), elaborada por Salmoni et al. (1984) e está relacionada ao fato de o CR guiar o individuo em relação à meta da tarefa. Porém, se for fornecido em altas frequências o mesmo pode gerar dependência da informação, negligenciado o processamento do feedback intrínseco, importante para o processo de aprendizagem, por fortalecer os mecanismos de detecção e correção dos erros. A segunda hipótese, denominada de consistência (consistency hypothesis) é baseada nos constantes ajustes realizados pelos indivíduos que recebem $\mathrm{CR}$ em altas frequências, ou seja, com menos $\mathrm{CR}$, o individuo realiza menos ajustes, tornando o desempenho na fase de aquisição mais estável (WINSTEIN; SCHMIDT, 1990).

Um volume grande de trabalhos foi conduzido com o objetivo de entender os efeitos de reduzir frequência de $C R$, sendo que é importante destacar que a manipulação da frequência de CR interage com o tipo de prática utilizada durante a fase de aquisição (LAl; SHEA, 1999; WULF; SHEA, 2004). A prática é considerada o fator mais importante para $O$ processo de aprendizagem motora (GUADAGNOLI; LEE, 2004), uma vez que, mesmo quando não é o fator a ser manipulado, ela está presente como meio para a manipulação de outros fatores (CORRÊA et al., 2006), dentre eles o CR.

Partido da interação entre prática e $\mathrm{CR}$, os efeitos de reduzir frequência de CR parecem ser verificados quando se utiliza de prática variada (WULF; SCHMIDT, 1989; SPARROW; SUMMERS , 1992; WULF; SHEA, 2004), que consiste na realização de uma variedade de versões de uma mesma tarefa motora, o que não ocorre quando se utiliza de prática constante (SPARROW; SUMMERS, 1992; LAI; SHEA, 1999; WULF; SHEA, 2004), onde os sujeitos executam apenas uma habilidade critério.

Chiviacowsky et al. (2009) ressalta que entre as variações da frequência de $C R$, uma delas tem sido reconhecida, mais recentemente, como relevante para a aprendizagem de habilidades motoras: o autocontrole. $\mathrm{O}$ autocontrole de $\mathrm{CR}$ na aprendizagem motora refere-se a um tipo de situação em que o aprendiz pode atuar mais ativamente no decorrer do processo, decidindo o melhor momento de receber informações relacionadas ao seu movimento (CHIVIACOWSKY; WULF, 2002). Se diferenciando das pesquisas realizadas até este momento na área da aprendizagem motora, onde Chiviacowsky et al. (2005) relata sobre um controle maior da prática por parte do pesquisador, enquanto pouca ou nenhuma ênfase é colocada no aprendiz, nas suas próprias estratégias de aprendizagem.

De acordo com Alcântara et al. (2007), a variável feedback extrínseco na abordagem autocontrolada não foi investigada em muitos estudos por ser uma área recente, porém aqueles realizados até o presente momento têm mostrado efeitos robustos para a aprendizagem de habilidades motoras. Os primeiros estudos sobre frequência autocontrolada de CR foram de Janelle et al. (1995) e Janelle et al. (1997), utilizando tarefas balísticas (tacada do golfe e arremesso de uma bola ao alvo com a mão não dominante, respectivamente) com prática constante. Janelle et al. (1995) comparou um grupo que recebeu frequência autocontrolada de CR em relação a outros quatro grupos que praticaram em diferentes condições: 1) $C R$ sumário a cada cinco tentativas; 2) $50 \%$ de frequência de $\mathrm{CR}$; 3) frequência de $\mathrm{CR}$ igual ao grupo autocontrolado, mas controlado pelo 
experimentador (também denominado em alguns estudos por grupo yoked); 4) grupo controle, que não recebeu $\mathrm{CR}$. Os resultados foram significativamente superiores para 0 grupo que recebeu $\mathrm{CR}$ com uma frequência autocontrolada comparado aos outros grupos. As vantagens do autocontrole de CR para a aprendizagem motora combinada a prática constante também foram descritas em vários outros estudos (WULF; TOOLE, 1999; CHIVIACOWSKY; WULF, 2002; CHIVIACOWSKY, et al. 2005; CHIVIACOWSKY, et al. 2006; ALCÂNTARA, et al. 2007; CHIVIACOWSKY, et al. 2008).

Os efeitos causadores deste benefício ainda não estão completamente esclarecidos, no entanto, Chiviacowsky e Wulf (2002) destacam três possíveis razões para as vantagens do $C R$ autocontrolado. A primeira razão é atender as necessidades específicas do aprendiz permitindo que ele decida em qual momento realmente necessita da informação. A segunda razão é encorajar 0 aprendiz a testar estratégias de movimento, o que o leva a tomar decisões e usar diferentes meios para regular sua própria aprendizagem; este fato foi observado no estudo de Chiviacowsky e Wulf (2002) que remete a preferência do aprendiz em receber o CR após a realização de "boas" tentativas ao invés das "más" tentativas de prática, a fim de confirmar seu desempenho em relação à meta. A terceira razão seria promover um processamento mais aprofundado de informações relevantes que, segundo Janelle et al. (1997), na psicologia educacional e cognitiva, estudos têm indicado que esse nível mais aprofundado de informações é alcançado através da participação ativa e independente do sujeito, utilizando estratégias autorreguladas para promoverem a retenção de informações cruciais.

Estudos a respeito da autorregulação do fornecimento de CR, geralmente, comparam um grupo autocontrolado a um yoked, também conhecido como grupo externamente controlado, em função das opções do grupo autocontrole. Em vários estudos (JANELLE et al., 1995; JANELLE et al., 1997; WULF; TOOLE, 1999; CHIVIACOWSKY; WULF, 2002; CHIVIACOWSKY, et al. 2005; CHIVIACOWSKY, et al. 2006; ALCÂNTARA et al., 2007; CHIVIACOWSKY et al., 2007; CHIVIACOWSKY et al., 2008; CHIVIACOWSKY et al., 2009; PETTERSON; CARTER, 2010; SIQUEIRA et al.,
2010) o grupo yoked recebe CR controlado pelo experimentador, onde os sujeitos deste grupo são equiparados um a um aos sujeitos do grupo autocontrolado, de forma que o número de CRs solicitados assim como o intervalo entre as solicitações são os mesmos para ambos os grupos, havendo um pareamento por tentativa.

No entanto, a forma de pareamento tradicional regula o processo de aprendizagem, baseado em outro sujeito (sujeito do grupo autocontrolado), ou seja, se o sujeito do grupo autocontrolado tem preferência em receber o CR após "boas" tentativas, a fim de confirmar seu desempenho em relação à meta (CHIVIACOWSKY; WULF, 2002), a mesma estratégia não ocorre com o sujeito pareado. Deste modo, é necessário investigar outras estratégias de parear o grupo autocontrolado, por exemplo, através da frequência média total de CRs, solicitados pelo grupo autocontrolado, sendo este valor médio transferido ao grupo yoked. A frequência média total de CRs mantém a característica de pareamento, por respeitar a quantidade total de informações solicitadas pelo grupo autocontrolado, mas distribuindo-as de forma mais equilibrada ao longo das tentativas de prática, para que o aprendiz tenha acesso à informação ao longo de todo o processo de aprendizagem. Deste modo, eliminando o efeito da regulação do processo de aprendizagem.

Ainda, percebe-se que os estudos sobre autocontrole de CR apresentam duas características: a utilização da prática constante na realização das tarefas motoras e o uso de um único formato de parear o grupo autocontrolado (por tentativa). Deste modo, faz-se necessário verificar os efeitos do autocontrole no fornecimento de CR na prática variada aleatória, além de investigar uma nova estratégia de constituir o grupo yoked. De acordo com Wulf e Toole (1999) a abordagem autocontrolada permite aos aprendizes se engajarem em atividades de processamento de informações diferentes daquelas dos grupos externamente controlados, exigindo uma maior demanda cognitiva. Shea e Morgan (1979) e Shea e Zimny (1983) relacionam a prática variada aleatória à elaboração e retenção na memória de todas as variações da habilidade que está sendo praticada. 
Com isso, surgem as duas questões que permearam este estudo: a aprendizagem autocontrolada apresentaria os efeitos benéficos, encontrados na prática constante, em um regime de prática variada aleatória? Poderia haver outra estratégia para parear o grupo autocontrolado? Assim, o objetivo do presente estudo foi analisar três diferentes estratégias de fornecer $\mathrm{CR}$, autocontrolado, yoked pareado por tentativa e yoked pareado pela frequência média total de $\mathrm{CR}$, na prática variada aleatória.

\section{Material e Métodos}

\section{Amostra}

Participaram do estudo 45 universitários de ambos os sexos e faixa etária entre 18 e 30 anos, selecionados em função da não experiência prévia na tarefa de pressionamento de teclas numéricas do computador com tempo alvo predeterminado. Os sujeitos não sofriam de incapacidades físicas ou mentais e não houve restrição de etnia, classes ou grupos sociais.

\section{Instrumentos e tarefa}

Foi utilizado um microcomputador, no qual os sujeitos utilizaram a região numérica do teclado para executar a tarefa e um software para controle das tentativas e armazenamento dos dados. Este software foi construído especificamente para estudos que utilizem a tarefa e o delineamento em questão, sendo utilizado pela literatura internacional (LAl; SHEA, 1998; 1999). A tarefa consistiu no pressionamento com o dedo indicador das teclas 2, 4, 8 e 6, nesta sequência, do teclado numérico do computador, em três diferentes tempos alvos absolutos (700, 900 e 1100 milissegundos), com prática variada aleatória. Essa tarefa foi escolhida por ser amplamente utilizada em pesquisas de aprendizagem motora (CHIVIACOWSKY; WULF, 2002; CHIVIACOWSKY et al., 2005; CHIVIACOWSKY et al., 2009; SANTOS et al., 2009; PATTERSON; CARTER, 2010).

\section{Delineamento experimental}

Os sujeitos foram distribuídos aleatoriamente em três grupos $(n=15)$ : GAC (autocontrolado), GY1 (yoked 1, pareado por tentativa, recebeu o CR exatamente nas mesmas tentativas do GAC) e GY2 (yoked 2, pareado pela frequência média total de CR).
O delineamento consistiu em três momentos: No primeiro momento foi realizada a fase de aquisição, com a execução de 72 tentativas (este número foi determinado após a realização de um estudo piloto, a fim de verificar o número de tentativas necessárias para alcançar a estabilização), com fornecimento de $\mathrm{CR}$ de acordo com cada grupo experimental. No segundo momento ocorreu o teste de retenção atrasado em vinte e quatro horas, com a execução de 9 tentativas, sem fornecimento de CR. No terceiro momento ocorreu o teste de transferência, também atrasado em vinte e quatro horas, logo após o teste de retenção, com a execução de 9 tentativas, sem fornecimento de CR.

$\mathrm{Na}$ fase de aquisição e no teste de retenção os três tempos alvo $(700,900,1100$ milissegundos) foram alterados aleatoriamente a cada tentativa, de modo que cada valor teve as mesmas chances de ser exibido. Os testes de retenção e transferência basearam-se na execução da tarefa em apenas um tempo alvo predeterminado que foi de $900 \mathrm{~ms}$ e $1300 \mathrm{~ms}$, respectivamente.

\section{Procedimentos experimentais}

O estudo foi aprovado pelo Comitê de Ética em Pesquisa da Universidade Federal dos Vales do Jequitinhonha e Mucuri, com o protocolo número 113/2010.

Os voluntários foram recrutados através de convites pessoais. Foi esclarecido desde o primeiro contato com os sujeitos que não haveria remuneração ou qualquer outro tipo de pagamento pela participação no estudo.

Ao ingressar no local da realização da coleta de dados, cada sujeito leu e, espontaneamente, assinou 0 termo de consentimento livre e esclarecido antes de iniciar os procedimentos experimentais.

No primeiro dia da coleta, o experimentador forneceu instruções a respeito da execução da tarefa e dinâmica da coleta dos dados através da leitura de um documento padrão. Este procedimento foi repetido para o segundo dia de coleta.

Durante a prática, os sujeitos deveriam sentarse de frente para uma mesa, em frente ao teclado numérico do computador e monitor. Certa 
liberdade no posicionamento do teclado foi concedida, a fim de manter um maior conforto e ajuste individual.

A coleta de dados foi realizada individualmente, em uma sala (segura e de fácil acesso) na Universidade Federal dos Vales do Jequitinhonha e Mucuri - UFVJM.

Os sujeitos que receberam $\mathrm{CR}$ autocontrolado foram informados que só deveriam solicitar o CR quando achassem que realmente precisariam do mesmo. Já os sujeitos do grupo com frequência externamente controlada receberam a informação de que às vezes receberiam a informação de $C R$ e às vezes não, mas que todas as tentativas eram importantes e seriam utilizadas para posterior análise.

Desta forma, os sujeitos do GAC receberam CR toda vez que solicitaram. Os sujeitos do GY1 foram pareados aos sujeitos do GAC, de forma que o número de CRs solicitados, assim como o espaçamento entre as solicitações, foram os mesmos para ambos os grupos. Já os sujeitos do GY2 também foram pareados aos sujeitos do GAC, porém foi realizada uma média geral da frequência de CRs solicitados por cada sujeito do GAC e esse valor médio foi transferido ao seu par do GY2 por bloco de nove tentativas. Por exemplo: um sujeito do GAC, em 72 tentativas, solicitou $50 \mathrm{CRs}$, ou seja, uma frequência de $69,44 \%$. Esse valor é transferido por bloco de 9 tentativas, correspondendo a 6,25 tentativas com CR. Assim, a cada 9 tentativas serão fornecidos 6 CRs (após arredondamento) ao sujeito do GY2 (ver Quadro 01). Além disso, assim como este exemplo, todas as situações de pareamento do GY2 seguiram o formato de distribuição conforme o quadro 1 abaixo:

Quadro 1. Distribuição de CRs pelos blocos de 9 tentativas do GY2.

\begin{tabular}{|cccccccccc|}
\hline № CRs & T1 & T2 & T3 & T4 & T5 & T6 & T7 & T8 & T9 \\
1 & & & & & X & & & & \\
2 & X & & & & X & & & & \\
3 & X & & & X & & & X & & \\
4 & & X & & X & & X & & X & \\
5 & X & & X & & X & & X & & X \\
6 & X & X & & X & X & & X & X & \\
7 & X & X & & X & X & X & & X & X \\
8 & X & X & X & X & & X & X & X & X \\
9 & X & X & X & X & X & X & X & X & X \\
\hline
\end{tabular}

\section{Análise estatística}

A medida utilizada neste estudo foi o erro absoluto (EA), ou seja, o valor, em milissegundos, da diferença entre o tempo realizado e o tempo predeterminado em cada tentativa.

Os valores do erro absoluto apresentados a cada tentativa, por voluntário, foram organizados pela média e desvio padrão em oito blocos de nove tentativas na fase de aquisição e um bloco de nove tentativas no teste de retenção e no teste de transferência. Para análise inferencial da média e desvio padrão do erro absoluto durante a fase de aquisição foi utilizado o teste ANOVA two way (3 grupos $X 9$ blocos) com medidas repetidas no segundo fator.

Para análise inferencial da média e desvio padrão do erro absoluto no teste de retenção e no teste de transferência foi utilizado o teste ANOVA one way ( 3 grupos $X 1$ bloco). O nível de significância adotado foi de $p<0,05$.

\section{Resultados}

Os resultados serão apresentados segundo as medidas utilizadas na análise dos dados (média e desvio padrão do erro absoluto) e discriminados conforme as fases do experimento (fase de aquisição, teste de retenção e teste de transferência).

\section{Média do erro absoluto \\ Fase de aquisição}

Os grupos mostraram desempenhos semelhantes com redução do erro do primeiro para o segundo bloco de tentativas e mantiveram o desempenho ao longo das demais tentativas (GRÁFICO 1, de A1 a A8).

A ANOVA two way ( 3 grupos $X 8$ blocos) com medidas repetidas no segundo fator não detectou diferença significante no fator grupos $[F(2,42)=$ $0,72, p=0,49]$ e na interação entre grupos e blocos $[F(14,294)=1,16, p=0,30]$. No entanto, foi encontrada diferença significante para 0 fator blocos $[F(7,294)=4,42, p=0,001]$, com o teste post hoc de Tukey indicando que $01^{\circ}$ bloco de tentativas apresentou desempenho inferior comparado aos demais blocos $(p<0,03)$.

\section{Teste de retenção}

Os grupos se comportaram de forma semelhante no teste de retenção (gráfico $1, T R$ ).

A ANOVA one way (3 grupos $X 1$ bloco) não detectou diferença significante no fator grupos $[F(2,42)=0,20, p=0,82]$. 


\section{Teste de transferência}

Os grupos se comportaram de forma semelhante no teste de transferência (gráfico 1, TR).
A ANOVA one way (3 grupos $X 1$ bloco) não detectou diferença significante no fator grupos $[F(2,42)=0,70, p=0,50]$.

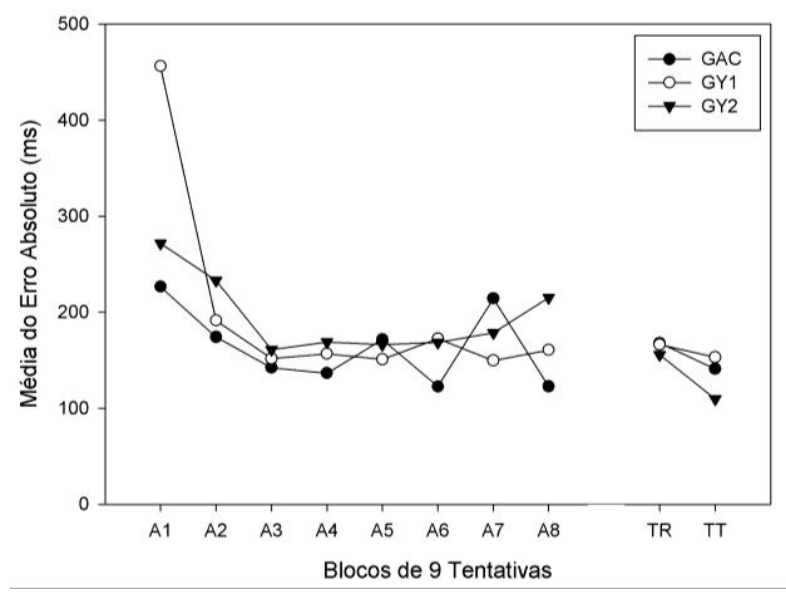

Gráfico 1. Média do EA dos três grupos experimentais durante a fase de aquisição e testes de retenção e transferência.

\section{Desvio padrão do erro absoluto Fase de aquisição}

Os grupos apresentaram desempenho semelhante, com redução da variabilidade do primeiro para o segundo bloco de tentativas, que se manteve ao longo das demais tentativas (gráfico 2, de $\mathrm{A} 1$ a $\mathrm{A} 8$ ).

A ANOVA two way ( 3 grupos $X 8$ blocos) com medidas repetidas no segundo fator não detectou diferença significante no fator grupos $[F(2,42)=$ $0,25, p=0,78]$ e na interação entre grupos $e$ blocos $[F(14,294)=1,61, p=0,07]$. Foi encontrada diferença significante para 0 fator blocos $[F(7,294)=2,39, p=0,02]$, com o teste post hoc de Tukey indicando que $01 \%$ bloco da fase de aquisição apresentou desempenho inferior comparado aos $3^{\circ}$ e $4^{\circ}$ blocos de tentativas $(p<$ $0,01)$.

\section{Teste de retenção}

Os grupos apresentaram um mesmo padrão de consistência durante a realização do teste de retenção (gráfico 2, TR).

A ANOVA one way ( 3 grupos $X 1$ bloco) não detectou diferença significante no fator grupos $[F(2,42)=0,51, p=0,60]$.

\section{Teste de transferência}

Os grupos apresentaram um mesmo padrão de consistência durante a realização do teste de transferência (gráfico 2, TR).

A ANOVA one way (3 grupos $X 1$ bloco) não detectou diferença significante no fator grupos $[F(2,42)=0,62, p=0,53]$.

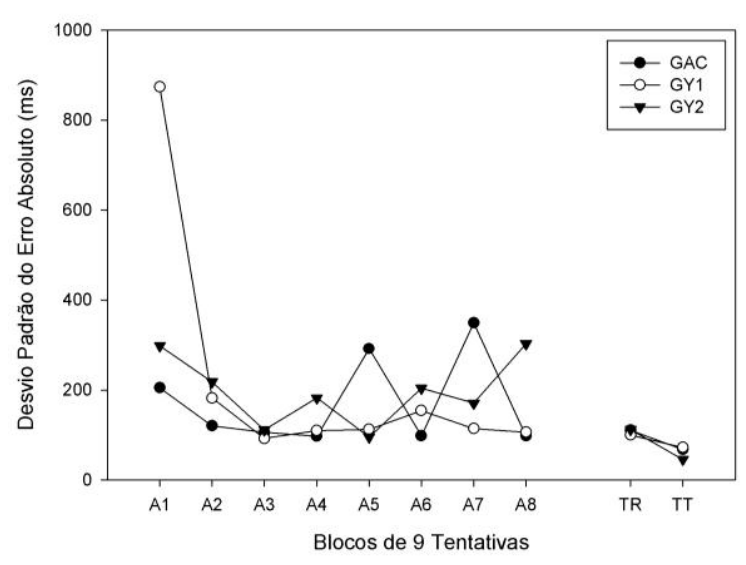

Gráfico 2. Desvio padrão do EA dos três grupos experimentais durante a fase de aquisição e testes de retenção e transferência. 


\section{Discussão}

O objetivo do presente estudo foi analisar três diferentes estratégias de fornecer $\mathrm{CR}$, autocontrolado e dois yoked, sendo esses pareados de formas distintas, em um arranjo de prática variada aleatória. Embora alguns estudos tenham demonstrado que a variável $\mathrm{CR}$ quando manipulada de forma autocontrolada tem alcançado resultados significativos na aprendizagem de habilidades motoras quando comparada a frequências controladas pelo experimentador (JANELLE, et al. 1995; JANELLE, et al. 1997), os resultados obtidos não apontaram diferenças significativas entre os grupos em nenhuma das fases avaliadas. Estudos que utilizaram delineamentos semelhantes, com grupos autocontrolado e yoked apontaram vantagens do primeiro grupo em relação ao segundo, mas não detectaram diferenças significativas (WULF; TOOLE, 1999; CHIVIACOWSKY; WULF, 2002; CHIVIACOWSKY et al., 2005; CHIVIACOWSKY, et al. 2006; ALCÂNTARA et al., 2007; CHIVIACOWSKY et al., 2008), nos testes de retenção e/ou transferência. Contudo, esses estudos utilizaram a prática constante.

Chiviacowsky et al. (2009), foi o único estudo encontrado que procurou investigar os efeitos da frequência autocontrolada de $\mathrm{CR}$ em um contexto de prática aleatória, apresentando características semelhantes ao do presente estudo, no que diz respeito ao tipo de instrumento utilizado, a tarefa e o tempo absoluto como uma das medidas de desempenho. Em ambos os trabalhos, os resultados não evidenciaram os efeitos benéficos do autocontrole na prática aleatória, sendo ainda obscuras as possíveis vantagens deste contexto.

Uma possível explicação para o fato de não encontrar diferenças pode estar relacionada ao CR ser influenciado por diversos fatores, dentre eles a prática (LAI; SHEA, 1999). Analisando os trabalhos conduzidos em relação à frequência relativa de $C R$, pode-se perceber que a literatura apresenta algumas divergências quanto ao efeito benéfico da redução da sua frequência relativa quando testada na prática constante (LAl; SHEA, 1999; WULF;SHEA, 2004). Por outro lado, quando é utilizada a prática seriada ou aleatória estudos têm observado efeito benéfico da frequência reduzida de CR (WULF; SCHMIDT, 1989; SPARROW; SUMMERS, 1992; WULF; SHEA, 2004).

Ainda, duas explicações podem contribuir para uma possível justificativa para a uniformidade observada no desempenho dos aprendizes neste estudo. A explicação de Wulf e Toole (1999) que defende a abordagem autocontrolada por permitir aos aprendizes se engajarem em atividades de processamento de informações, exigindo uma maior demanda cognitiva. E a explicação de Shea e Morgan (1979) e Shea e Zimny (1983) que defendem a prática variada aleatória, por permitir ao aprendiz comparar e distinguir na memória de trabalho todas as variações do movimento. Ambas as explicações ao invés de evidenciarem os benefícios da prática autocontrolada em um arranjo aleatório, conforme apontado na introdução deste artigo, acabaram por justificar os resultados aqui encontrados, principalmente pela amostra se tratar de sujeitos iniciantes na tarefa em questão. Assim, segundo Chiviacowsky et al. (2009), as altas cargas de processamento de informações empregados nas tarefas autorreguladas juntamente com a sobrecarga da memória de trabalho provenientes da prática aleatória podem ter dificultado a elaboração de estratégias relacionadas à melhoria do desempenho, o que pode ter levado a equidade entre os grupos estudados.

Ainda, utilizando a proposição teórica de Guadagnoli e Lee (2004) que demonstram que existe a necessidade de uma quantidade ótima informação, que varia em função do nível de habilidade do aprendiz bem como da dificuldade da tarefa a ser aprendida, a combinação entre a prática aleatória e o feedback autocontrolado pode ter gerado um aumento da dificuldade nominal (reflete a condição na qual a tarefa é desempenhada) modificando os efeitos das variáveis experimentais. Deste modo, apesar de a literatura demonstrar o efeito individual de cada uma das variáveis independentes quando manipuladas separadamente, quando duas 
variáveis são combinadas um efeito diferente do comportamento individual pode ser encontrado (TANI et al., 2006). Este fato pode ter ocorrido com os resultados do presente estudo. Todavia novos estudos precisam ser conduzidos para uma confirmação destes resultados.

Referente a utilização de uma única forma de parear o grupo autocontrolado, todos os estudos utilizaram apenas o pareamento por tentativa. $O$ presente estudo não nega os resultados das pesquisas anteriores no que diz respeito à constituição do grupo yoked, apenas apresenta outra possibilidade de compor este grupo, já que ambas as formas de pareamento, por tentativa e pela frequência média de $C R$, apresentaram desempenho semelhante durante as duas fases do experimento.

Em suma, os resultados encontrados no presente estudo demonstram que houve aprendizagem devido à melhoria no desempenho, via redução do erro, porém de forma semelhante entre os grupos experimentais, o que não contradiz os efeitos benéficos da aprendizagem autocontrolada.

\section{Conclusão}

Os resultados levam a concluir que não houve efeito da frequência autocontroladas de CR para aprendizagem motora quando um arranjo de prática variada aleatória é utilizado. Além disso, o estudo permitiu uma nova possibilidade de parear o grupo autocontrolado, através da frequência média total de $C R$, já que este apresentou os mesmos benefícios para a aprendizagem do pareamento por tentativas.

Seria interessante que outros estudos fossem realizados utilizando o autocontrole de $\mathrm{CR}$ em diferentes regimes de prática, sobretudo na prática variada aleatória, a fim de obter resultados consistentes que possibilitem um melhor entendimento do autocontrole de CR na aquisição de habilidades motoras.

\section{Referências}

ADAMS, J.A. A closed-loop theory of motor learning. Journal of Motor Behavior, Washington, v. 3, n. 2, p. 111-149, 1971.
ALCÂNTARA, L.B.; ALVES, M.A.F.; SANTOS, R.C.O.; MEDEIROS, L.K.; GONÇALVES, W.R.; FIALHO, J.V.A.P.; UGRINOWITSCH, H.; BENDA, R.N. Efeito do conhecimento de resultados autocontrolado na aprendizagem de habilidades motoras em idosos. Brazilian Journal of Motor Behavior, Belo Horizonte, v. 2, n. 1 p. 22-30, 2007.

BILODEAU, E.A.; BILOUDEAU, I.M. Variable frequency of knowledge of results and the learning on acquisition and extinction of a positioning task. Journal of Experimental Psychology, Washington, v. 55, n. 4, p. 379-383, 1958.

CHIVIACOWSKY, S.; KAEFER, A.; MEDEIROS, F.L.; PEREIRA, F.M. Aprendizagem motora em crianças: "feedback após boas tentativas melhora a aprendizagem? Revista Brasileira de Educação Física e Esporte, São Paulo, v. 21, n. 2, p. 157-165, 2007.

CHIVIACOWSKY, S.; MEDEIROS, F.L.; KAEFER, A. "Feedback" auto-controlado e aprendizagem de uma tarefa motora com demanda de força. Revista Brasileira de Educação Física e Esporte, São Paulo, v. 21, n. 1, p. 27-33, 2007.

CHIVIACOWSKY, S.; MEDEIROS, F.L.; SCHILD, J.F.G.; AFONSO, M.R. Feedback autocontrolado e aprendizagem de uma habilidade motora discreta em idosos. Revista Portuguesa de Ciências do Desporto, Porto, v. 6, n. 3, p. 275280, 2006.

CHIVIACOWSKY, S.; NEVES C.; LOCATELLI L.; OLIVEIRA C. Aprendizagem motora em crianças: efeitos da frequência autocontrolada de conhecimentos de resultados. Revista Brasileira de Ciências do Esporte, Campinas, v. 26, p. 177-190, 2005.

CHIVIACOWSKY, S.; PINHO, S.T.; ALVES, D.; SCHILD, J.F.G. "Feedback" autocontrolado: efeitos na aprendizagem de uma habilidade específica do golfe. Revista Brasileira de Educação Física e Esporte, São Paulo, v. 22, n. 4, p. 265-271, 2008.

CHIVIACOWSKY, S.; TREPTOW, J.G.; TANI, G.; MEIRA JUNIOR, C.; SCHILD, J.F.G. Conhecimento de resultados autocontrolado: efeitos na aprendizagem de diferentes programas motores generalizados. Revista Portuguesa de Ciências do Desporto, Porto, v. 9, n. 2+3, p. 175-182, 2009.

CHIVIACOWSKY, S.; WULF, G. Self-controlled feedback: does it enhance learning because performance get feedback when they need it? 
Research Quarterly for Exercise and Sport, Washington, v. 73, p. 408-415, 2002.

CORRÊA, U. C.; GONÇALVES, L. A.; BARROS, J. A. C.; MASSIGLI, M. Prática constantealeatória e aprendizagem motora: efeitos da quantidade de prática constante e da manipulação da exigência da tarefa. Brazilian Journal of Motor Behavior, Belo Horizonte, v. 1, n. 1, p. 41-52, 2006.

GUADAGNOLI, M.A.; LEE, T.D. Challenge point: a framework for conceptualizing the effects of various practice conditions in motor learning. Journal of Motor Behavior, Washington, v.36, p.212-24, 2004.

JANELLE, C.M.; BARBA, D.A.; FREHLICH, S.G.; TENNANT, L.K.; CAURAUGH, H. Maximizing performance feedback effectiveness through videotape replay and a self-controlled learning environment. Research Quarterly for Exercise and Sport, Washington, v. 68, p. 269-279, 1997.

JANELLE, C.M.; KIM, J.; SINGER, R.N. Subjectcontrolled performance feedback and learning of a close motor skill acquisition. Perceptual and Motor Skills, Missoula, v. 81, p. 627-634, 1995.

LAI, Q.; SHEA, C.H. Generalized motor program (GMP) learning: effects of reduced frequency of knowledge of results and practice variability. Journal of Motor Behavior, Washington, v. 30, n.1, p. 51-59, 1998.

LAI, Q.; SHEA, C.H. The role of reduced frequency of Knowledge of results during constant practice. Research Quarterly for Exercise and Sport, Washington, v. 70, n. 1, p. 33-40, 1999.

MANOEL, E.J. A dinâmica do estudo do comportamento motor. Revista Paulista de Educação Física e Esporte, v.13, n. especial, São Paulo, p. 52-61, 1999.

OLIVEIRA, D. L.; CORRÊA, U. C.; GIMENEZ, R.; BASSO, L.; TANI, G. Relative frequency of knowledge of results and task complexity in the motor skill acquisition. Perceptual and Motor Skills, Missoula, v. 109, n. 3, p. 831-840, 2009.

PATTERSON, J.T.; CARTER, M. Learner regulated knowledge of results during the acquisition of multiple timing goals. Human Movement Science, Amsterdam, v. 29, p. 214227, 2010.

SALMONI, A.W., SCHMIDT, R.A.; WALTER, C.B. Knowledge of results and motor learning: a review and critical reappraisal. Psychological Bulletin, Washington, v. 95, n. 3, p. 355-386, 1984.
SANTOS, R.C.O.; LAGE, G.M.; UGRINOWITSCH, H.; BENDA, R.N. Efeitos de diferentes proporções de prática constante e aleatória na aquisição de habilidades motoras. Revista Brasileira de Educação Física e Esporte, São Paulo, v. 23, n. 1, p. 5-14, 2009.

SCHMIDT, R.A. A schema theory of discrete motor skill learning. Psychological Review, Washington, v. 82, n. 4, p. 225-260, 1975.

SCHMIDT, R.A.; LEE, T. D. Motor Control and Learning: A behavioral emphasis. 4.ed. Champaign: Human Kinetics, 2005.

SHEA, J.B.; MORGAN, R.L. Contextual interference effects on the aquisition, retention and transfer of a motor skill. Journal of Experimental Psychology: Human Learning and Memory, Washington, v. 5, n. 2, p. 179-187, 1979.

SHEA, J.B.; ZIMNY, S.T. Context effects in memory and learning movement information. In: MAGGIL, R.A. (Ed.) Memory and Control of Action. Amsterdam: North Holland, 1983, p. 345366.

SIQUEIRA, A.K.M.; HENRIQUE, R.S.; BELTRÃO, N.B.; CATTUZZO, M.T. Efeito do autocontrole de conhecimento de resultados na aquisição de uma habilidade motora. Revista da Educação Física, Maringá, v. 21, n. 4, 2010.

SPARROW, W.A.; SUMMERS, J.J. Performance on trials without knowledge of results $(K R)$ in reduced relative frequency presentations of KR. Journal of Motor Behavior, Washington, v. 24, n. 2, p. 197-209, 1992.

TANI, G.; SANTOS, S.; MEIRA JUNIOR, C. M . $O$ ensino da técnica e aquisição de habilidades motoras no desporto. In: TANI, G.; BENTO, J. O.; PETERSEN, R. D. S. (Eds.). Pedagogia do Desporto. Rio de Janeiro: Guanabara-Koogan, 2006. p. 227-240.

WINSTEIN, C.J.; SCHMIDT, R.A. Reduced frequency of knowledge of results enhances motor skill learning. Journal of Experimental Psychology: Learning, Memory and Cognition, Washington, v. 16, n. 4, p. 677-691, 1990.

WULF, G.; SCHMIDT, R.A. The learning of generalized motor programs: reducing the relative frequency of knowledge of results enhances memory. Journal of Experimental Psychology: Learning, Memory and Cognition, Washington, v. 15 , n. 4 , p. $748-757,1989$.

WULF, G.; SHEA, C.H. Understanding the role of augmented feedback: The good, the bad, and the ugly. In: WILLIAMS A.M.; HODGES, N.J. Skill 
acquisition in sport: Research, theory and

practice. London: Routledge, 2004, p. 121-144.

WULF, G.; TOOLE, T. Physical assistance devices in complex motor skill learning: benefits of a self-controlled practice schedule. Research Quarterly for Exercise and Sport, Washington, v. 70, p. 265-272, 1999.

\section{Endereço:}

Gabriela Moara Ferreira

Av. Silvio Felício dos Santos, 1120 Bom Jesus

Diamantina MG Brasil

39100-000

Telefone: (38) 3531-5498 e (38) 8822-4566

e-mail: gabrielamoara@yahoo.com.br

Recebido em: 5 de abril de 2011.

Aceito em: 4 de abril de 2012.

\section{(a) (1)}

Motriz. Revista de Educação Física. UNESP, Rio Claro, SP,

Brasil - elSSN: 1980-6574 - está licenciada sob Creative

Commons - Atribuição 3.0 